\title{
A FLOW SOURCE FOR THE STUDY OF QUANTUM TURBULENCE IN SUPERFLUID ${ }^{4} \mathrm{HE}$
}

\author{
Simone Babuin ${ }^{1}$, Miloš Rotter ${ }^{2}$, Mathias Stammeier ${ }^{3}$, Josef Šebek ${ }^{1}$, Ladislav Skrbek ${ }^{2}$ \\ Abstract: A flow source has been developed to generate turbulent superfluid flows \\ in channels equipped for detection of quantised vortices. The source consists of a \\ motor driven low temperature compressible volume capable of pushing or sucking \\ superfluid ${ }^{4} \mathrm{He}$ into a flow channel. Detection is based on the attenuation of second \\ sound caused by the presence of quantised vortex lines in the turbulent flow. In this \\ paper we present the technical details of the apparatus, along with some raw data \\ obtained during the first successful low temperature test. The test has been \\ performed using the source to generate a flow into a channel whose ends have \\ been blocked by superleaks to allow for the inflow and outflow of the superfluid \\ component of helium only. This technique enables to determine the areal density of \\ quantised vortex lines in the temperature range 1.3 to $2.1 \mathrm{~K}$, with well controlled \\ flow velocities between 0.01 and $30 \mathrm{~cm} / \mathrm{s}$.
}

\section{INTRODUCTION}

Quantum turbulence is the particular type of turbulence which occurs in quantum fluids i.e. fluids whose hydrodynamics is partly determined by quantum mechanical effects. In this paper we are concerned with the superfluidity of liquid ${ }^{4} \mathrm{He}$ and its ability to undergo turbulent motion. The purpose of the paper is to describe a novel technique for the generation of turbulent superfluid flows, to review a method for their quantitative detection, and to present some preliminary raw data to demonstrate the ability of the technique to enable interesting experiments. Before a presentation of the flow source, a brief introduction to superfluid hydrodynamics and quantum turbulence is now in order, to provide the reader with the basic background [1] necessary to appreciate the type of measurements possible with this technique.

Liquid ${ }^{4} \mathrm{He}$, between its boiling point at temperature $\mathrm{T}=4.2 \mathrm{~K}$ and $\mathrm{T}=2.17 \mathrm{~K}$ is an ordinary Navier-Stokes fluid of very low kinematic viscosity. Below $\mathrm{T}=2.17 \mathrm{~K}$ the liquid undergoes a phase transition to a different state, He-II, which persists down to $\mathrm{T}=0 \mathrm{~K}$. He-II can be phenomenologically described as a perfect mixture of two fluids, an ordinary viscous fluid with viscosity and entropy content, and an ideal inviscid fluid with no viscosity and no entropy. These two fluids, called respectively normal and superfluid components, are interpenetrating and capable of sustaining independent motion if their relative velocity is small, of order of $\mathrm{cm} / \mathrm{s}$, whilst at higher velocities coupling between them becomes possible. The densities of the two components, $\rho_{n}$ and $\rho_{s}$, are such that $\rho_{n}+\rho_{s}=\rho$, where $\rho$ is the total density of helium. The ratio $\rho_{s} / \rho$ grows monotonically with decreasing temperature: from zero at the transition, to unity at $\mathrm{T}=0 \mathrm{~K}$.

\footnotetext{
${ }^{1}$ Institute of Physics ASCR, v.v.i., Na Slovance 2, 18221 Prague, Czech Republic

${ }^{2}$ Faculty of Mathematics and Physics, Charles University, Ke Karlovu 3, 12116 Prague, Czech Republic

${ }^{3}$ Ruprecht-Karls-University, Seminarstraße 2, 69120 Heidelberg, Germany
} 
We are interested in the ability of this two-fluid system to sustain developed turbulence. The type of turbulence which can be supported by the superfluid is determined by its quantum nature. In the superfluid a macroscopically large fraction of atoms occupies the same quantum ground state. This state is described by a wavefunction whose amplitude squared represents the superfluid density and whose phase, S, is related to its dynamics. It can be easily shown that the superfluid velocity, $v_{s}$, is proportional to the gradient of the wave-function's phase, $\boldsymbol{v}_{s}=\hbar \nabla \mathrm{S} / \mathrm{m}$, where $\hbar$ is the reduced Plank's constant and $m$ the mass of one ${ }^{4} \mathrm{He}$ atom. From here one can immediately deduce that curl $\boldsymbol{v}_{s}=0$, hence the circulation of the velocity around a closed loop in the fluid, $\Gamma=\oint \boldsymbol{v}_{s} \cdot d \boldsymbol{l}$, is identically zero, upon application of Stokes theorem of calculus and provided that the loop encloses a simply connected portion of fluid. This would signify that the superfluid cannot be set to rotary motion by accelerating the walls of a container where it rests. However, experimental evidence shows that this is not the case: a superfluid in a rotating container displays classical-like solid-body-rotation, with a free surface parabolic profile and circulation $\Gamma=2 \Omega$, where $\Omega$ is the angular velocity of the container. A non-zero circulation is possible and it is consistent with the condition $\operatorname{curl} \boldsymbol{v}_{s}=0$ because the superfluid under rotation generates vortices, i.e. zero curl rotary motion around thin vortex cores, or lines, where superfluidity is suppressed. The circulation calculated around a loop which encloses a vortex line - and therefore an area where the superfluid is not simply connected - is given by

$$
\Gamma=\oint \boldsymbol{v}_{S} \cdot d \boldsymbol{l}=\frac{\hbar}{m} \oint \boldsymbol{\nabla S} \cdot d \boldsymbol{l}=\frac{\hbar}{m} 2 \pi n=n \kappa,
$$

which follows by noticing that the change of phase over a full turn around the loop must be an integer multiple of $2 \pi$, for the superfluid wave function has a unique value at any given point in space. The circulation is therefore a non-zero quantity, quantised in units of the quantum of circulation $\kappa=h / m=9.9 \times 10^{-8} \mathrm{~m}^{2} / \mathrm{s}$, and curl $\boldsymbol{v}_{s}$ is zero everywhere except in the vortex cores.

For the simple case of superfluid rotation in a cylindrical container rotating about its vertical axis, vortex lines appear parallel to the axis of rotation, and each vortex contributes to the global rotation with one quantum of circulation. The circulation per unit area is therefore equal to $\kappa L$, where $L$ is the number of vortex lines per unit area, or equivalently, the average vortex line length per unit volume. A vortex line is the essential constituent of a turbulent superfluid flow.

For a generalised flow, vortex lines will constitute a disordered tangle. Vortex lines originate from - and end to - the walls of the container (or free surface, if there is one), or close up on themselves to form loops. These structures may interact with each other and with the normal component of He-II, and may undergo complex dynamics whose discussion is beyond the scope of this article. An introduction to vortex dynamics can be found in [2] and some recent direct visualization of vortex lines is reported in [3].

Typically, quantum turbulence experiments are designed to measure the number of vortex lines per unit area, $L$, although the generation of turbulence and its detection may vary. Broadly speaking, for superfluid ${ }^{4} \mathrm{He}$, there are two classes of flow generation: classical (as for ordinary viscous fluids) and non-classical. Among classically generated flows we list counter-rotating blades [4], towing of grids [5] and the rapid spin down of a rotating container $[6,7,8]$. Non-classical flows are flows for which no classical analogue exists, namely the ability of the normal and superfluid components of He-II to move relative to each other. This relative motion, known as counterflow, occurs, for example, when a heater is placed in a bath of He-II causing the normal component to move away from it to transport entropy and the superfluid to move towards it to conserve mass. Thermal counterflows have been historically the first flows to be investigated in quantum turbulence $[9,10]$. Pure superflow, a variety of counterflow where the motion of the normal component is impeded, has also been studied $[10,11]$. 


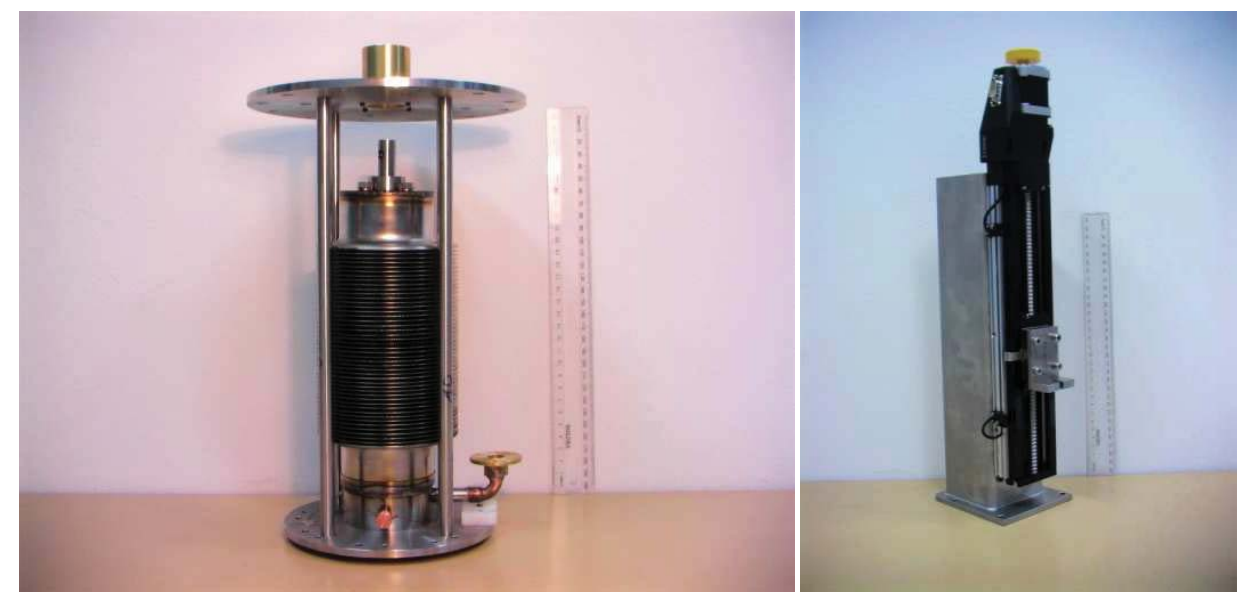

Figure 1. (left) The low temperature bellows before installation to the cryostat. The pin at the top of the bellows is the connection to the vertical shaft driven by a motor which pulls and compresses the bellows. The flow outlet to the bottom right connects to the flow channel. (right) Programmable stepper motor before installation to the cryostat. The motor is installed on top of the cryostat at room temperature and drives the shaft connected to the bellows. In both photographs the ruler is $30 \mathrm{~cm}$ long.

The flow source described in this article belongs to the classical flow generation category. It is based on compressing a low temperature volume containing He-II to push fluid into channels where it can be detected. The flow source is versatile and it can be used to generate flows into a variety of channels. However, in this paper we describe one type of flow only, obtained with a channel designed so as to allow inflow and outflow of the superfluid component only. In this sense, although the manner in which the flow is generated is, so to speak, classical, the type of flow produced is not.

In the next section the experimental setup is presented whilst in section 3 some examples of measurements are provided.

\section{THE FLOW SOURCE AND ITS EXPERIMENTAL CONTEXT}

The essence of our technique is to employ a low temperature bellows, i.e. a compressible volume residing in a He-II bath to controllably push/suck fluid into/from a flow channel equipped for the detection of quantised vortex lines.

Our home-built low temperature bellows - before its installation to the cryostat - is shown in the left photograph in Figure 1. It consists of a vertical stuck of stainless steel layers welded on top of each other and forming a hollow volume with one outlet (bottom right in the photo) where flow channels can be connected. In Figure 2 the bellows is photographed in its final assembly located at the bottom of the insert of a standard open bath ${ }^{4} \mathrm{He}$ cryostat, also built in-house for this purpose. The bellows is moved by a programmable motor (shown on the right hand side of Figure 1) mounted at room temperature on the stainless steel flange of the cryostat and connected to the bellows via a vertical shaft which runs along the central axis of the cryostat.

A leak-tight seal against the moving shaft is made by spring loaded rubber ' $O$ '-rings. The motor is a Micos VT-75 2SM 2-phase stepper motor with $1.2 \mathrm{~A}$ phase current and holding torque of $350 \mathrm{mNm}$. It is capable to effect precise vertical positioning with a resolution of $20 \mu \mathrm{m}$ and can move the bellows with speed controlled to within about $2 \%$, depending on the magnitude of velocity and load. Movement commands are given by an external PC with dedicated software.

To the left of the bellows in Figure 2 one can see a vertical flow channel. This channel has been used in the present work for testing the new apparatus and perform preliminary 
measurements of vortex lines density. The very same channel was used in a previous experiment where the flow was instead driven thermally by a helium fountain pump [11]. The reader is referred to that work for a full description of the channel and the sensors for detection of vortex lines. At any rate, a brief description of the apparatus shall be given here for convenience.

The brass flow channel has internal square cross-section of $7 \times 7 \mathrm{~mm}^{2}$ and length of about $100 \mathrm{~mm}$. At both ends of the channel there is a brass cylinder containing a silver sintered superleak, i.e. a roughly $50 \%$ open tight packing of silver spheres with average inter-sphere gap in the sub $\mu \mathrm{m}$ range. The purpose of the superleak is to block the inflow of the normal (viscous) component of helium into the channel and therefore generate a pure superflow. The normal component of helium is viscously locked into a porous medium as long as its viscous penetration depth is larger than the pore size, a condition easily satisfied for our flows.

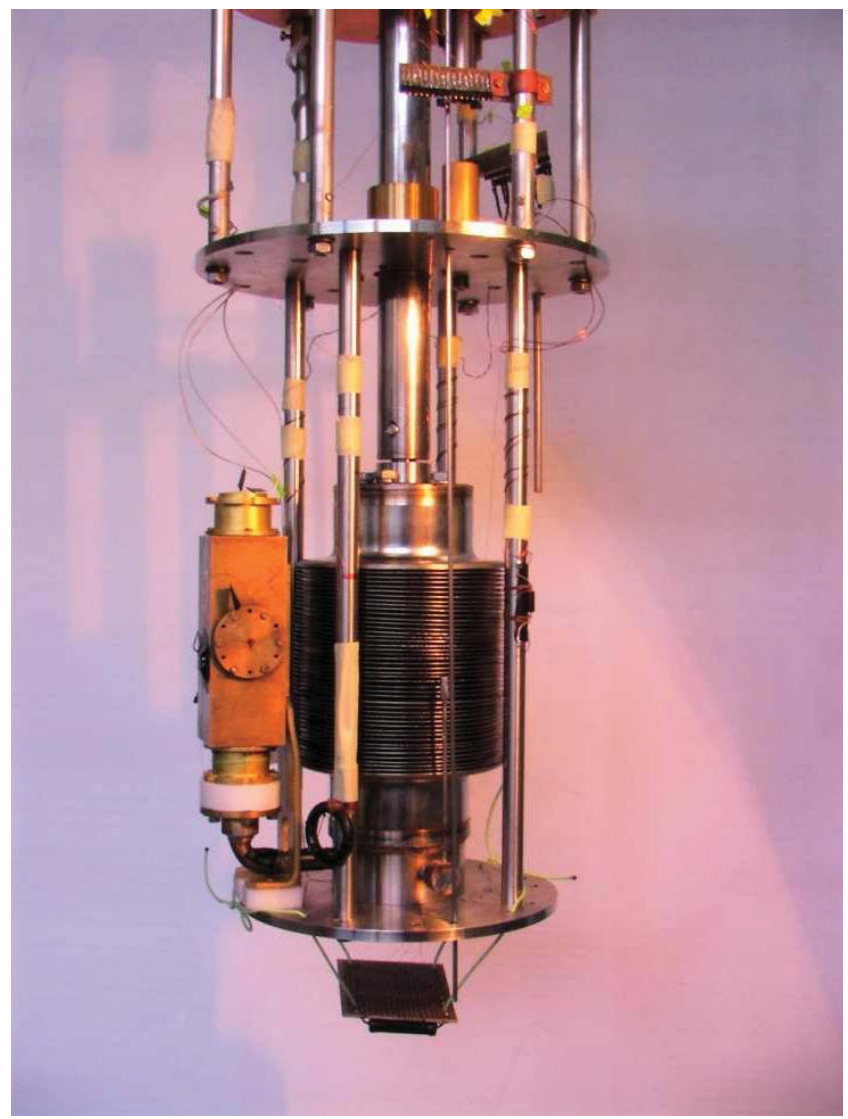

Figure 2. Photograph of the low temperature bellows in its final assembly. The bellows hangs at the bottom of the ${ }^{4} \mathrm{He}$ open bath cryostat insert. To the left of the bellows is the vertical flow channel. The channel has silver sinter superleaks inside brass cylinders at its ends and second sound sensors located at half of its height, enclosed behind the circular lid. The wiring feeds thermometers and heaters for temperature control.

Two brass circular blocks located at half channel height (one visible in the photo) contain sensors for the detection of superfluid turbulence. Detection is based on the attenuation of second sound transmitted transversally to the flow direction.

Second sound is a temperature wave which originates from the counter-oscillation of normal and superfluid components [1]. In our experiment second sound is generated by AC vibration of a plastic membrane dotted with micropores. The idea is that motion of the membrane against the fluid displaces the normal component which does not see the micron-size holes, whilst the inviscid superfluid component remains at rest. This causes a 
pressure wave in the normal component, immediately cancelled by motion of the superfluid to keep the overall helium density constant. Second sound standing waves can be set up in the transverse direction of the channel, which acts as a resonator. An identical membrane located at the other side of the channel acts as a second sound receiver.

The speaker membrane moves because it forms parts of a capacitor. One plate of the capacitor is a golden plated side of the membrane which faces the interior of the channel, whilst the other plate is represented by a spring-loaded electrode pressed against the other side of the membrane. An Agilent 33220A function generator supplies AC sinusoidal voltage to the capacitor causing alternated attraction and repulsion between the plates, hence membrane motion. Sound excitation is most efficient if the gold-plated side of the capacitor is electrically lifted from ground by $100 \mathrm{~V}$. For this reason the whole channel is insulated from the cryostat by a Delrin plastic buffer located between the bottom superleak and the bellows outlet, also visible in the photograph.

The receiver side works similarly, except that here a mechanical membrane motion caused by second sound produces an AC voltage response in the capacitor. This signal is detected by a Stanford SR830 lock-in amplifier.

The essence of our detection technique is that, when turbulent superfluid flow is generated in the channel, the presence of quantised vortex lines causes extra second sound attenuation relative to the attenuation observed when $\mathrm{He}$-II is at rest in the channel. The attenuation is caused by scattering of sound waves off the vortex cores [12]. This effect is quantifiable by measuring the reduction of the amplitude of second sound resonance detected with the lock-in amplifier. This point shall be developed further in the next section where sample resonator data are presented.

To complete the description of the setup, our prime thermometry is provided by the ${ }^{4} \mathrm{He}$ saturated vapour pressure measured with a MKS Baratron 690A pressure gauge. The temperature of the experiment is varied and controlled by adjusting the pumping rate on the cryostat helium bath by means of a traditional in-series combination of rotary and roots vacuum pumps.

Three carbon resistor thermometers are located inside the bellows, equally spaced on a stick along the vertical axis. These sensors work simultaneously as liquid level meters and actual thermometers to monitor the change of temperature inside the bellows upon compression and expansion. The thermometers are read by a Neocera LTC-21 temperature controller.

External to the bellows, a thermometer and heater wired to the temperature controller are available if finer temperature control than what provided by adjusting the pumping rate should be needed.

\section{DETERMINATION OF FLOW VELOCITY, MEASUREMENT PROCEDURES, AND EXAMPLES OF RAW DATA}

A typical aim of this class of quantum turbulence experiments based on channel flow is to measure the density of vortex lines, $L$, as a function of flow velocity. In the present work the flow velocity is the velocity of the superfluid component, $\boldsymbol{v}_{s}$, owing to the presence of the superleaks.

The idea is to assume the incompressibility of He-II [1] and determine the flow velocity from the conservation of helium mass flowing from the compressed bellows into the channel. If $V$ is the bellows volume and $A$ the cross-sectional area of the channel ( $7 \times 7$ $\mathrm{mm}^{2}$ in our case), then we have

$$
\frac{d V}{d t}=A v_{s}
$$

We then rewrite the left hand side of (2) as 


$$
\frac{d V}{d t}=\frac{d V}{d l} \frac{d l}{d t^{\prime}}
$$

where $l$ is the vertical coordinate to measure bellows displacement, and therefore $v_{b}=d l / d t$ is the speed at which the bellows is moved. By substituting (3) into (2) we get

$$
v_{s}=\frac{1}{A} \frac{d V}{d l} v_{b} .
$$

which directly converts the velocity of the bellows into the velocity of the flow in the channel. The quantity $d V / d l$ is obtained from a calibration of bellows volume against its vertical displacement, or extent of compression, so to speak. The bellows vertical displacement is known directly by accurately measuring the vertical position of the shaft connected to the motor, using the motor's encoder. The bellows volume cannot be determined geometrically in a simple way, because of its complicated zigzag shape. We therefore used a method based on the accurate measurement of the pressure drop observed when a certain amount of helium gas contained in the bellows is allowed to expand into an external volume of known size.

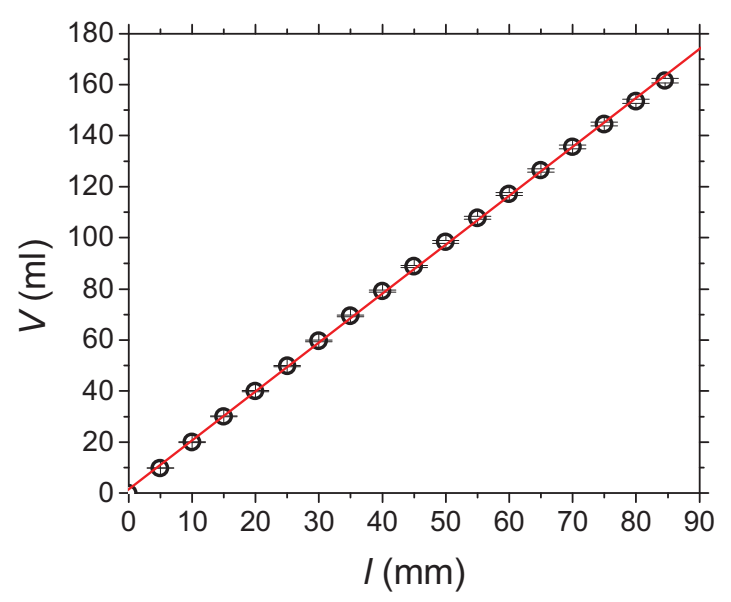

Figure 3. Calibration of bellows volume, $V$, against bellows vertical position, $I$. The origin of the graph corresponds to the bellows in its most stretched position. Increasing $I$ indicates compression of the bellows and the volume measured is the volume reduction with respect to the fully stretched position. The straight line is a linear fit with a slope $d V / d l=1.91 \pm 0.01 \mathrm{ml} / \mathrm{mm}$.

The calibration results are shown in Figure 3, where the vertical displacement of the bellows ranges from the fully stretched to the fully compressed positions. The linear fit returns a slope $d V / d l=1.91 \pm 0.01 \mathrm{ml} / \mathrm{mm}$. The (small) uncertainty on the slope, together with the uncertainty on $A$ and on $v_{b}$ (the latter depends weakly on the magnitude of the motor velocity and on the temperature of He-II being pushed against the channel's superleak in the actual experiment) results in our ability to determine the superfluid speed, $v_{s}$, with a precision between about $1.5 \%$ and $3 \%$.

The typical procedure when running an experiment is to start with both bellows and flow channel fully immersed in a bath of He-II, at the desired temperature. The bellows is kept at first in the fully stretched position and is completely filled with He-II. A second sound resonance is set up across the channel, and by monitoring the time variation of the maximum amplitude at resonance, a statistically steady state is waited for. When the 
conditions in the channel are stable with the fluid at rest (instabilities might have arisen because of previous bellows movements, for example) a flow of desired velocity is generated. Flows are only generated by compression of the bellows, not extension. The reason for this is that, since the helium bath is pumped and has a low pressure of order of mbar, the flows generated by suction of He-II from the bath have a velocity which depends on the pressure head due to the helium height difference between the bath and the channel. This pressure head decreases with the drop of helium level in the bath, therefore determination of superflow speed in case of suction is less accurate.

In Figure 4 we show some typical examples of raw resonator data, for different flow speeds and different temperatures. In the left panel one can see the modification of the second sound resonant peak caused by a flow of velocity $9.6 \mathrm{~cm} / \mathrm{s}$ at a temperature of $1.34 \mathrm{~K}$. The signal shown is the quadrature component of the speaker response amplified by the lock-in amplifier. The open circles represent the signal in case of no flow. A Lorentzian curve fit (solid line in the Figure) returns an amplitude of $1.76 \mathrm{mV}$ and a full width at half maximum of $38.3 \mathrm{~Hz}$. The effect of the flow is to change these values to $1.25 \mathrm{mV}$ and $53.1 \mathrm{~Hz}$, respectively. There is no change in the position of the central frequency, to within the resolution of the fit.

On the right panel of Figure 4 we plot the maximum of the resonant peak amplitude as a function of time (step-shaped line with values on left vertical axis) at $\mathrm{T}=1.65 \mathrm{~K}$. This trace is produced by exciting sound at the fixed resonant frequency and recording in time the amplitude of the response. The dashed line indicates the displacement of the bellows, with values reported on the right vertical axis (a position of $0 \mathrm{~mm}$ corresponds to the bellows being fully stretched). At $\mathrm{t}=0 \mathrm{~s}$ there is no flow in the channel and the steady state amplitude is of about $1 \mathrm{mV}$. At about $\mathrm{t}=20 \mathrm{~s}$ the bellows is compressed with a velocity of $1.2 \mathrm{~cm} / \mathrm{s}$, corresponding to a flow speed of $4.7 \mathrm{~cm} / \mathrm{s}$. The bellows is compressed almost instantaneously on the time scale of the plot, with an acceleration of $30 \mathrm{~cm} / \mathrm{s}^{2}$. It can be seen that the amplitude signal drops sharply to about $0.65 \mathrm{mV}$ after which a steady state is quickly established. At about $t=40 \mathrm{~s}$ the motion of the bellows is halted and the signal recovers the no-flow value, after an (exponential) transient. The second step in the trace is simply a repetition of the same procedure, compressing further the bellows, and for a slightly longer time. Notice that the technique easily resolves this $35 \%$ amplitude suppression, with a good signal to noise ratio of about 30 , which is fairly typical except for very slow speeds where it worsens by a factor of 10 or so.

We have collected similar data in the temperature range $1.34 \mathrm{~K}$ to $2.1 \mathrm{~K}$, and for flow velocities ranging from about $0.01 \mathrm{~cm} / \mathrm{s}$ to $30 \mathrm{~cm} / \mathrm{s}$, but only the two examples in Figure 4 are shown here for brevity.

In a previous experiment [11] which used the same flow channel employed in the present work, the flow was driven by the helium fountain effect [1]. A heater was attached just above the top superleak and the whole channel was moved with an adjustable positioning system so that the heater was always kept just below the surface of the helium bath. When the heater is switched on, the superfluid component rushes towards it - owing to counterflow - passing through the bottom and top superleaks, and thus generating a superflow in the channel. The outgoing superflow produces a fountain of He-II at the top of the channel. In [11] the superfluid speed was calculated with an analytic formula obtained by demanding conservation of superfluid mass in and out of the channel. This calculation required assuming, among other things, that all the heat delivered by the fountain heater is used to converting superfluid into normal component and that the superfluid and normal component velocities are matched in the fountain stream, therefore neglecting the possibility of counterflow between the bath and the heater via the fountain stream (see [11] for details on the calculation).

One advantage of the present technique is that it removes the need of such assumptions for the determination of flow velocity, allowing much better velocity control especially at very low flow velocities. 

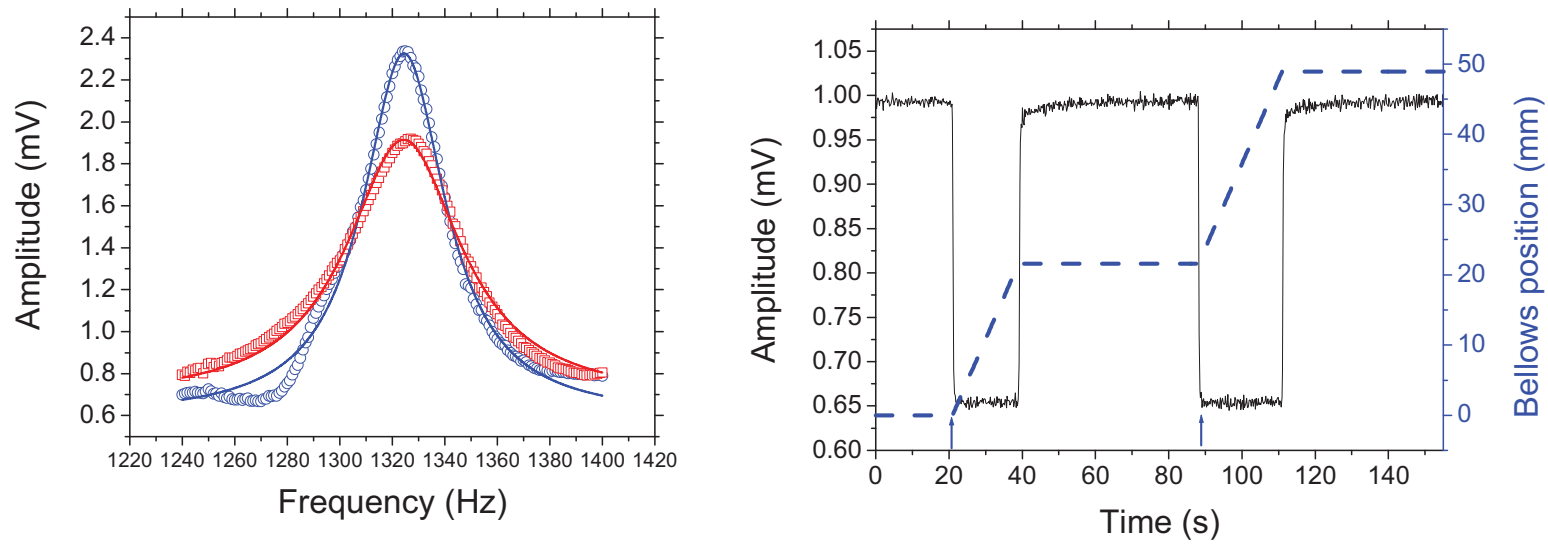

Figure 4. Examples of suppression of second sound amplitude caused by turbulent flow in the channel. (left) Full second sound resonant peak for stationary fluid in the channel (open circles) and for a flow speed of $9.6 \mathrm{~cm} / \mathrm{s}$ (open squares), at $\mathrm{T}=1.34 \mathrm{~K}$. The lines are a Lorentzian curve fit to the data. The fit produces an amplitude of $1.76 \mathrm{mV}$ and full width at half maximum of $38.3 \mathrm{~Hz}$ in the case of no flow. These values change to $1.25 \mathrm{mV}$ and $53.1 \mathrm{~Hz}$ respectively when turbulent superflow is generated. The central frequency of the peak is not modified by the flow. (right) Amplitude of the second sound resonant curve at maximum plotted as a function of time, at $T=1.65 \mathrm{~K}$. The arrows at about $20 \mathrm{~s}$ and $90 \mathrm{~s}$ indicate the time when the bellows is compressed with a constant speed of $1.2 \mathrm{~cm} / \mathrm{s}$, corresponding to a flow speed of $4.7 \mathrm{~cm} / \mathrm{s}$. The dashed line indicates the bellows vertical displacement from the fully stretched position (position values on right axis). The suppression of second sound amplitude is clearly visible in the solid line trace.

Another advantage is the ability to set fluid to motion in the channel virtually instantaneously, due to the very high accelerations afforded by the motor, and not achievable by the helium fountain method where the fastness of response of flow to heat dissipation is intrinsically limited by the thermodynamic nature of the process.

The resonator data presented in Figure 4 can be used for the determination of the number of vortex lines per unit area, $L$, under the assumption that the tangle of vortex lines formed inside the channel is homogeneous and isotropic [12]. If this is the case, then $L$ can be calculated data as follows:

$$
L=\frac{6 \pi W_{0}}{\kappa B}\left(\frac{A_{0}}{A}-1\right),
$$

where $A_{0}$ and $A$ are the second sound resonance amplitudes respectively without and with flow, $W_{0}$ is the full width at half maximum of resonance in case of no flow, and $B$ is the mutual friction coefficient, a tabulated value related to the intensity of interaction between normal component and vortex lines [2].

A systematic study of the dependence of the vortex lines density with superfluid speed, at different temperatures, is currently under way.

\section{Conclusion}

In this article we have presented a new experimental apparatus for the generation and detection of a turbulent flow in superfluid helium. The apparatus is based on a low temperature bellows, i.e. a compressible volume filled with superfluid helium which 
pushes a flow of very well determined velocity into a channel. The turbulence generated in the channel is in the form of a tangle of quantised vortex lines, whose areal density can be measured by the observation of the extra attenuation of second sound resonances set up transversally to the flow direction.

In the test run presented here the channel was $10 \mathrm{~cm}$ long, $0.7 \mathrm{~cm}$ wide with square cross-section and had both ends blocked with superleak to allow for inflow and outflow of the superfluid component of helium only. The test was successful and good quality second sound attenuation data were obtained in the flow velocity range 0.01 to $30 \mathrm{~cm} / \mathrm{s}$ and temperature range 1.3 to $2.1 \mathrm{~K}$.

The merit of this technique over the thermal flow generation used in previous works is the ability to calculate the flow velocity to better than $3 \%$ accuracy for all flow conditions and without requiring thermodynamic assumptions. Furthermore, the technique allows to impose very large accelerations to the fluid and to obtain good velocity control also at very small flow velocities.

The bellows flow source is versatile and can be utilised in combination with channels of different dimensions and features, thus allowing quantum turbulence studies to be performed on different types of flow.

\section{ACKNOWLEDGMENTS}

We thank Marco La Mantia and David Schmoranzer for useful discussion on apparatus development and help in the laboratory with construction and data collection.

We acknowledge the contribution of the Institute of Scientific Instruments of the ASCR, v.v.i. in Brno, Czech Republic, for the construction of the bellows.

We thank Tymofiy V. Chagovets for earlier development work on the cryostat, the flow channel, and software for resonator data collection.

We acknowledge support from research grants GAČR \#202/08/0276 and AVOZ 10100520.

\section{REFERENCES}

[1] Tilley D. R. and Tilley J.: Superfluidity and superconductivity, Institute of Physics Publishing, Bristol, 1988

[2] Vinen W. F. and Niemela J. J.: Quantum turbulence, J. Low Temp. Phys. 128, 2002, 167-231

[3] Bewley G. P., Lathrop D. P. and Sreenivasan K. R.: Visualization of quantised vortices, Nature 441, 2006, 588

[4] Maurer J. and Tabeling P.: Local investigation of superfuid turbulence, Europhys. Lett. 43, 1998, 29

[5] Stalp S. R., Skrbek L. and Donnelly R. J.: Decay of grid turbulence in a finite channel, Phys. Rev. Lett. 82, 1999, 4831

[6] Walmsley P. M., Golov A. I., Hall H. E., Levchenko A. A. and Vinen W. F.:

Dissipation of quantum turbulence in the zero temperature limit, Phys. Rev. Lett. 99, 2007, 265302.

[7] Walmsley P. M., Golov A. I.: Quantum and quasiclassical types of superfuid turbulence, Phys. Rev. Lett. 100, 2008, 245301

[8] Walmsley P. M., Golov A. I., Hall H. E., Vinen W. F. and Levchenko A. A.: Decay of turbulence generated by spin-down to rest in superfuid He-4, J. Low Temp. Phys.

153, 2008, 127

[9] W. F. Vinen: Mutual friction in a heat current in liquid helium II

I. Experiments on steady heat currents, Proc. Roy. Soc. A 240, 1957, 114

II. Experiments on transient effects, Proc. Roy. Soc. A 240, 1957, 128

III. Theory of the mutual friction, Proc. Roy. Soc. A 242, 1957, 493 
IV. Critical heat currents in wide channels, Proc. Roy. Soc. A 243, 1958, 400

[10] Tough J. T.: Superfluid turbulence, in Prog. in Low Temp. Phys. vol. VIII, NorthHolland, Amsterdam, 1982

[11] Chagovets T. V. and Skrbek L.: On flow of He II in channels with ends blocked by superleaks, J. Low Temp. Phys. 153, 2008, 162-188

[12] Barenghi C. F., Gordeev A. V., Skrbek L.: Depolarization of decaying counterflow turbulence in He-II, Phys. Rev. E 74, 2006, 026309 